\title{
Accurate diagnosis of axillary lymph node metastasis using contrast-enhanced ultrasonography with Sonazoid
}

\author{
FUMIHIKO MATSUZAWA $^{1}$, TAKAHIRO EINAMA ${ }^{1}$, HIRONORI ABE ${ }^{1}$, TAKASHI SUZUKI ${ }^{1}$, \\ JUN HAMAGUCHI ${ }^{1}$, TERUMI KAGA ${ }^{2}$, MAMI SATO ${ }^{2}$, MASAKO OOMURA ${ }^{2}$, YUMIKO TAKATA ${ }^{2}$, \\ AYAKO FUJIBE $^{2}$, CHIE TAKEDA ${ }^{2}$, ETSUYA TAMURA $^{2}$, AKINOBU TAKETOMI $^{3}$ and KENICHI KYUNO ${ }^{1}$ \\ Departments of ${ }^{1}$ Surgery and ${ }^{2}$ Clinical Laboratory, Hokkaido Social Work Association \\ Obihiro Hospital, Obihiro, Hokkaido 080-0805; ${ }^{3}$ Department of Gastroenterological Surgery I, \\ Hokkaido University Graduate School of Medicine, Sapporo, Hokkaido 060-8638, Japan
}

Received October 30, 2014; Accepted December 16, 2014

DOI: $10.3892 /$ mco.2014.483

\begin{abstract}
Axillary lymph node enlargement following sentinel lymph node biopsy (SLNB) is often difficult to accurately diagnose. In keeping with the characteristically tortuous and aberrant pattern of tumor neovasculature, metastatic lymph nodes exhibit peripheral and mixed vascularity, resulting in a microvasculature that is often difficult to visualize. Contrast-enhanced ultrasonography (CEUS) with Sonazoid, a new generation contrast agent for ultrasonography, allows for the visualization of lymph node microvessels and may enable a more accurate evaluation of lymph node metastasis. This is a case report of axillary lymph node enlargement following SLNB, in which CEUS with Sonazoid resulted in an accurate diagnosis. On the basis of our experience with this case, we have initiated a clinical trial to evaluate the detection of lymph node metastasis through the use of CEUS in breast cancer patients.
\end{abstract}

\section{Introduction}

Sentinel lymph node biopsy (SLNB) has become an alternative to axillary lymph node dissection (ALND) for nodal staging in breast cancer $(1,2)$. If the sentinel lymph node (SLN) is free of metastasis, metastatic disease is not likely to be present in the axillary lymph nodes; therefore, ALND may be avoided (3). However, SLNB has been reported to be associated with false-negative results and local recurrence following SLNB (4).

In keeping with the characteristically tortuous and aberrant pattern of tumor neovasculature, metastatic lymph nodes also exhibit peripheral and mixed vascularity. Color Doppler ultrasonography only provides information

Correspondence to: Dr Takahiro Einama, Department of Surgery, Hokkaido Social Work Association Obihiro Hospital, 2 East 5 South 9, Obihiro, Hokkaido 080-0805, Japan

E-mail: titiuehahaue@hotmail.com

Key words: Sonazoid, lymph node swelling, sentinel lymph node biopsy regarding macrovessel flow and morphology; therefore, it is difficult to accurately diagnose lymph node metastasis using this method (5). Sonazoid, a new generation contrast agent for ultrasonography, allows for visualization of lymph node microvessels. Compared to previously used imaging modalities, contrast-enhanced ultrasonography (CEUS) with Sonazoid may enable a more accurate evaluation of lymph node metastasis.

This is a case report of axillary lymph node metastasis (ALNM) following SLNB, which was accurately diagnosed with CEUS using Sonazoid.

\section{Case report}

A 40-year-old woman underwent total mastectomy and SLNB for cancer of the left breast. The histopathological examination revealed invasive ductal carcinoma, T1bNOM0, stage IA, estrogen receptor-negative, progesterone receptor-negative, human epidermal growth factor receptor-negative and MIB-1 index $6 \%$. The patient received tamoxifen as adjuvant endocrine therapy. After 6 months, follow-up enhanced computed tomography (CT) revealed a left axillary lymph node enlarged to $11 \mathrm{~mm}$ (Fig. 1A). Color Doppler ultrasonography revealed pulsatile blood flow to the lymph node from several directions (Fig. 1B). On the basis of the CT and color Doppler results, lymph node metastasis was suspected. In contrast to the color Doppler, CEUS demonstrated blood flow from only the hilum of the lymph node, suggesting that this lymph node may be clear of metastasis (Fig. 2). ALND was ultimately performed and the histopathological findings confirmed that the axillary lymph node was negative for metastasis (Fig. 3).

This study was approved by the Research Ethics Committee of our hospital and the patient provided consent for the findings of her case to be published.

\section{Discussion}

Breast cancer is the most common type of cancer in women, exhibiting considerably varying incidence and mortality rates worldwide. The 5-year survival rate in patients with breast cancer reportedly ranges between 74 and $82 \%$ (6). 

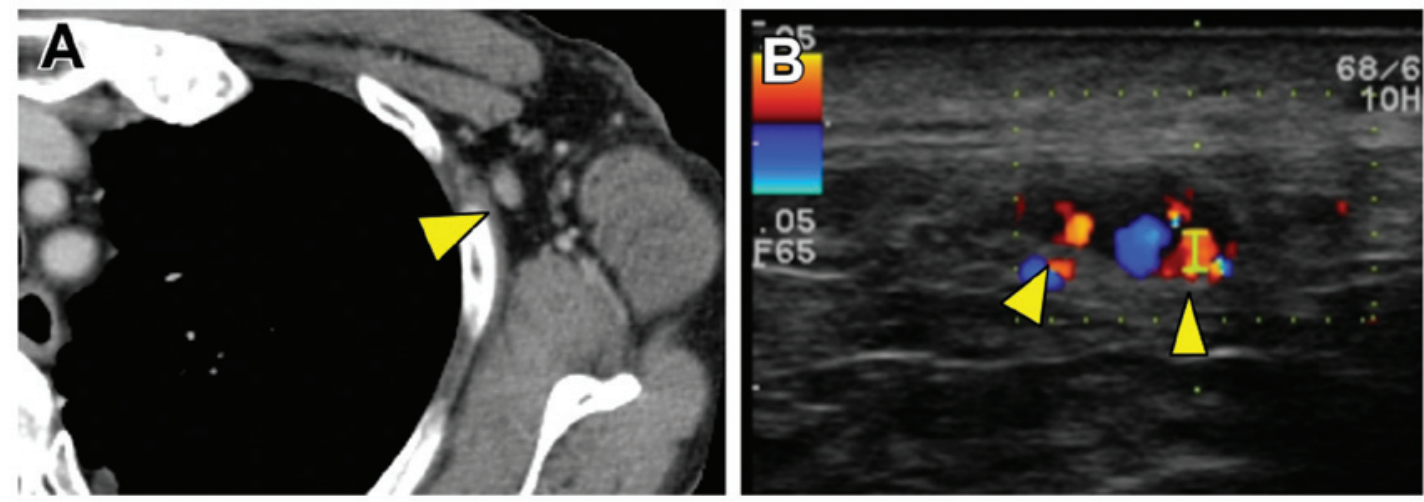

Figure 1. (A) Contrast-enhanced computed tomography revealed an enhanced enlarged lymph node (arrowhead). (B) Color Doppler ultrasonography revealed pulsatile blood flow (arrowheads) to the lymph node from several directions.
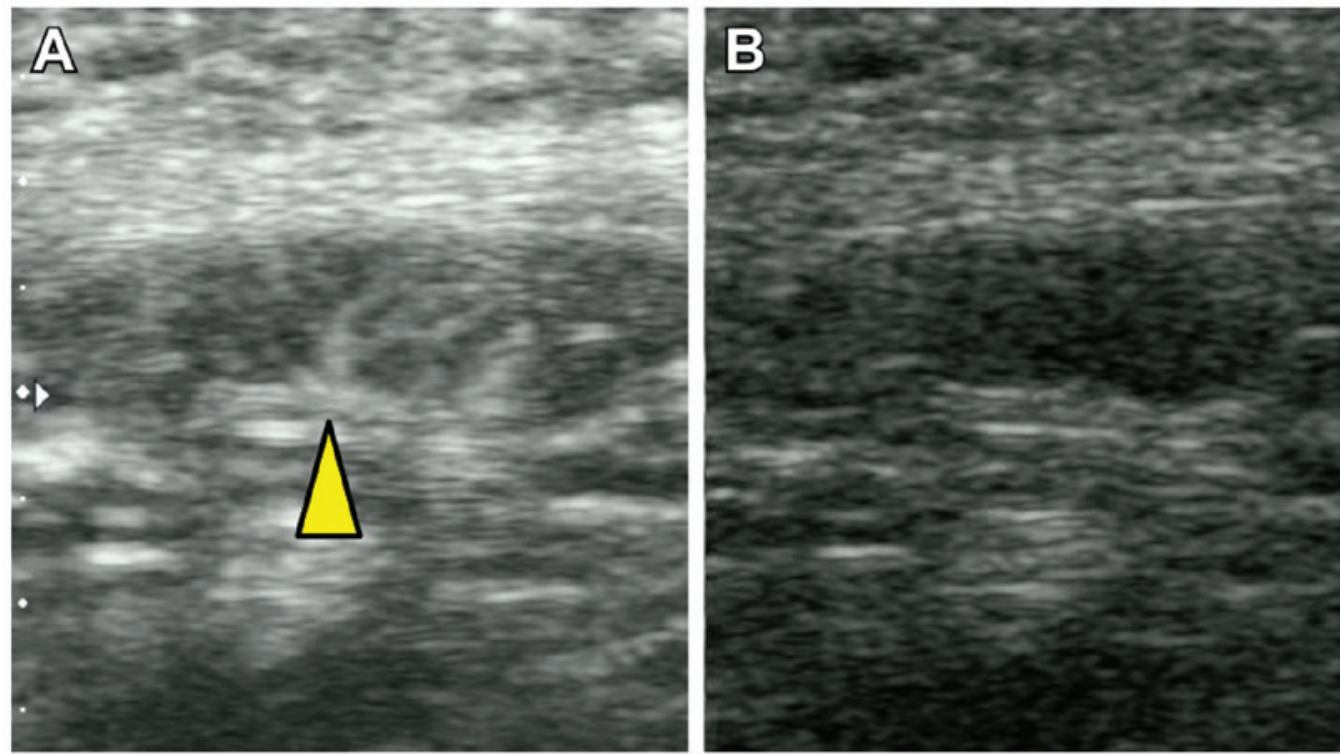

Figure 2. (A) Contrast-enhanced ultrasonography with Sonazoid demonstrated blood flow from only the hilum of the lymph node (arrowhead). (B) Micro Flow Imaging, B-mode.
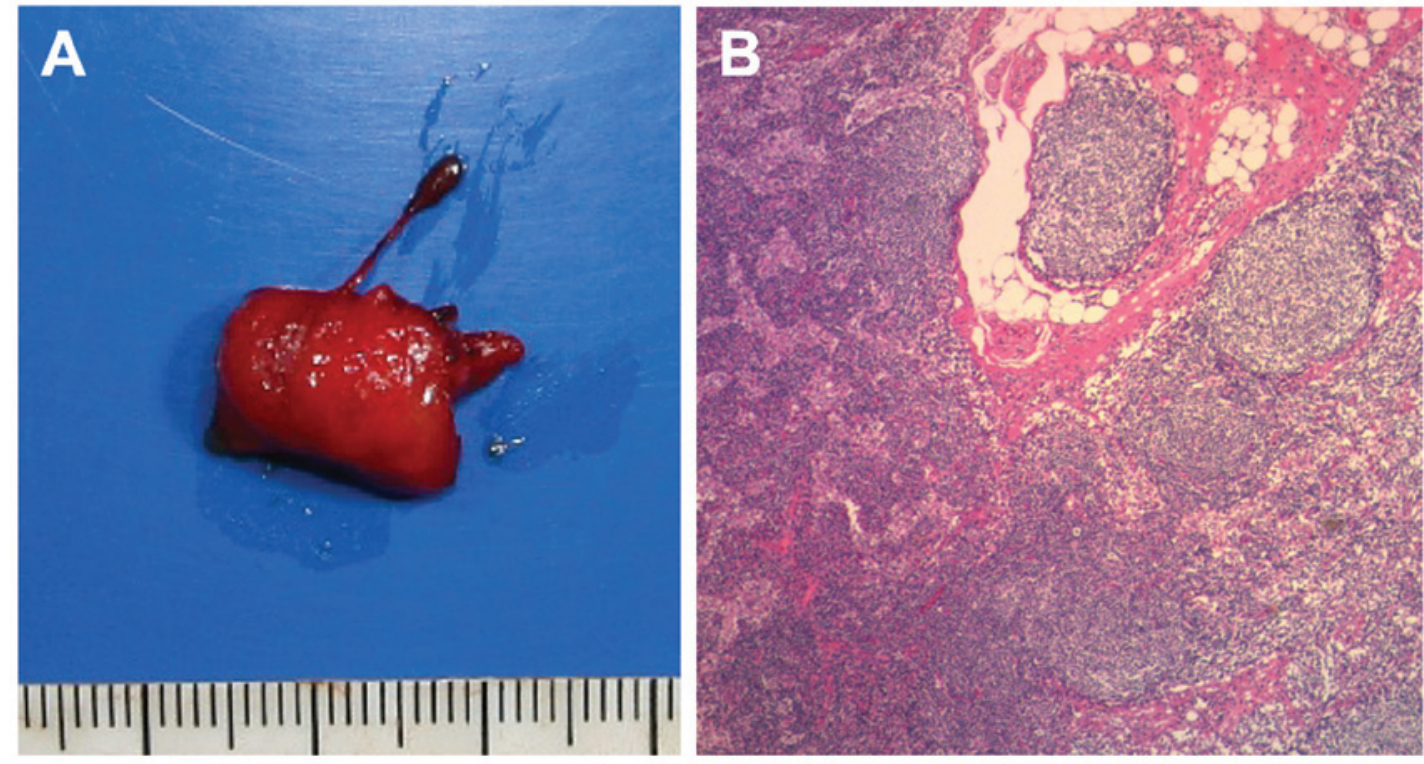

Figure 3. The resected lymph node was not malignant. (A) Gross appearance of the resected lymph node. (B) Histopathological examination of the resected lymph node, which was negative for breast cancer metastasis (hematoxylin and eosin-stained tissue; magnification, x20). 
Table I. Comparison of results of axillary lymph node metastasis detection by CT, Doppler US and CEUS.

\begin{tabular}{|c|c|c|c|c|}
\hline Case no. & CT & US (with Doppler) & CEUS & Pathological findings \\
\hline 1 & Negative & Negative & Negative & Negative \\
\hline 2 & Negative & Negative & Negative & Negative \\
\hline 3 & Negative & Positive & Positive & Positive \\
\hline 4 & Negative & Negative & Negative & Negative \\
\hline 5 & Negative & Negative & Negative & Negative \\
\hline 6 & Negative & Negative & Positive & Positive \\
\hline 7 & Positive & Negative & Negative & Negative \\
\hline 8 & Negative & Negative & Negative & Negative \\
\hline 9 & Negative & Negative & Negative & Negative \\
\hline 10 & Negative & Negative & Negative & Negative \\
\hline 11 & Negative & Negative & Negative & Negative \\
\hline 12 & Negative & Negative & Negative & Negative \\
\hline 13 & Negative & Negative & Positive & Positive \\
\hline Present case & Positive & Positive & Negative & Negative \\
\hline Total, (\%) & $10 / 14(71)$ & 11/14 (79) & $14 / 14(100)$ & - \\
\hline
\end{tabular}

CT, computed tomography; US, ultrasonography; CEUS, contrast-enhanced US.

Since standard radical mastectomy for the treatment of breast cancer was first established by Halsted, the surgical procedures for breast cancer have continued to improve on the basis of the results of randomized clinical trials (2,7-11). Breast-conserving surgery is now considered to be the standard local treatment for early breast cancer. In addition, SLNB has recently become an alternative to ALND for nodal staging $(1,2)$.

The SLN hypothesis states that tumor cells that are shed from a primary carcinoma migrate through a lymphatic channel to a single lymph node prior to involvement of the remaining lymph nodes within that basin. The SLN is the first lymph node that receives lymphatic drainage from a tumor and its identification and analysis for tumor involvement may predict the status of the remaining lymph nodes (12).

However, several issues have been reported when using SLNB for nodal staging. First, it was reported that the proportion of patients with successfully mapped SLNs ranged between 41 and $100 \%$, with $>50 \%$ of the studies reporting a rate $<90 \%$. The false-negative rate ranged between 0 and $29 \%$, with an average rate of $7.3 \%$ (13). Second, patients may experience local disease recurrence following SLNB. Axillary local recurrence rates in patients with a negative SLNB and no ALND were reported to range between 0 and $1.4 \%$ at 14-46 months of follow-up (4).

ALNM is a key factor for the prognosis of breast cancer and significantly affects the decisions regarding the selection of treatment modalities; thus, diagnostically accurate methods for determining ALNM are crucial. Axillary ultrasonography (AUS) is widely used for the detection of ALNM, as it is relatively accurate and non-invasive. AUS is simple, easy and more cost-effective compared to other modalities. Therefore, it is an elemental test in breast cancer evaluation. The sensitivity and specificity of AUS for the detection of ALNM were reported to be 61 and $82 \%$, respectively (14). Contrast-enhanced magnetic resonance imaging (cMRI) is generally used to evaluate the regional extent of breast cancer prior to breast-conserving surgery; it enables the assessment of the changes in the extent of tumor growth pre- and post-chemotherapy and may be used for screening of high-risk patients and of those with large breasts, evaluating isolated ALNMs of unknown origin and evaluating ALNMs in breast cancer $(15,16)$. The sensitivity and specificity of cMRI for the prediction of ALNM range between $36-100 \%$ and 54-100\%, respectively. These ranges are fairly wide, as they are dependent on the definition of ALNM, the type of contrast agent used, the size of the breast tumor and the number of metastatic ALNs (17-21). Hwang et al (22) reported that the actual accuracy of cMRI was similar to that of AUS. Imaging with fluoro-2-deoxy-D-glucose-positron emission tomography (PET) may also be used to evaluate ALNM. The fundamental strength of PET imaging over conventional imaging is its ability to convey functional information that even the most exquisitely detailed anatomical image cannot provide. However, when surveyed across the multitude of prior reports, PET has an overall sensitivity of $88 \%$, a specificity of $92 \%$ and an accuracy of $89 \%$, although several of the studies achieved a higher sensitivity at the expense of lower specificity or vice versa. This may explain the wide variation in the results (23).

Sonazoid, a new generation contrast agent for ultrasonography, was first introduced on January 10, 2007 and is approved for use only in Japan. The active ingredient of Sonazoid is a perflubutane microbubble that is stabilized using hydrogenated egg phosphatidyl serine sodium, which is a phospholipid. Perflubutane is chemically stable and insoluble in water. Therefore, it has a long lifespan in the body, as it hardly dissolves in the blood. CEUS with Sonazoid for liver tumors is currently frequently performed in Japan. Omoto et al (24) reported an SNL detection method using CEUS with Sonazoid in a human breast cancer patient. Aoki et al (25) suggested that 
CEUS may be useful in distinguishing tumor-induced from inflammation-induced lymph node enlargement.

CEUS with Sonazoid may also allow for the visualization of microvessels. Color Doppler ultrasonography provides information on macrovessel flow and morphology and may evaluate palpable lymph nodes more accurately compared to AUS; however, it cannot be used to evaluate microvessels and is therefore not applicable in the evaluation of non-palpable nodes (5). We suggest that CEUS with Sonazoid may enable an accurate diagnosis of ALNM.

In our department, we have performed preoperative evaluation using CEUS for lymph node metastasis in a total of 14 cases (Table I). In addition, we also performed plane ultrasonography, color Doppler ultrasonography, enhanced CT and enhanced MRI during the preoperative examination. An accurate diagnosis using CEUS was possible in all the cases. In cases 6 and 13, as well as in the present case, the results differed between CEUS and the other imaging modalities. Considering these results, CEUS is more likely to result in an accurate diagnosis of lymph node metastasis compared to other modalities used for evaluation.

In conclusion, we presented a case with axillary lymph node enlargement following SLNB, in which CEUS with Sonazoid resulted in an accurate diagnosis. On the basis of our experience with this case, a clinical trial evaluating the detection of lymph node metastasis by CEUS in breast cancer patients is currently ongoing at our institution.

\section{Acknowledgements}

The authors would like to thank E. Tamura, M. Satoh, T. Kaga, M. Ohmura, Y. Takada, C. Takeda and A. Fujibe for making this study possible.

\section{References}

1. Lyman GH, Giuliano AE, Somerfield MR, et al; American Society of Clinical Oncology: American Society of Clinical Oncology guideline recommendations for sentinel lymph node biopsy in early-stage breast cancer. J Clin Oncol 23: 7703-7720, 2005.

2. McCready D, Holloway C, Shelley W, et al; Breast Cancer Disease Site Group of Cancer Care; Ontario's Program in Evidence-Based Care: Surgical management of early stage invasive breast cancer: a practice guideline. Can J Surg 48: 185-194, 2005.

3. Dabakuyo TS, Fraisse J, Causeret S, et al: A multicenter cohort study to compare quality of life in breast cancer patients according to sentinel lymph node biopsy or axillary lymph node dissection. Ann Oncol 20: 1352-1361, 2009.

4. Naik AM, Fey J, Gemignani M, et al: The risk of axillary relapse after sentinel lymph node biopsy for breast cancer is comparable with that of axillary lymph node dissection: a follow-up study of 4008 procedures. Ann Surg 240: 462-471, 2004.

5. Yang WT, Chang J and Metreweli C: Patients with breast cancer: differences in color Doppler flow and gray-scale US features of benign and malignant axillary lymph nodes. Radiology 215: $568-573,2000$

6. Parkin DM, Bray F, Ferlay J and Pisani P: Global cancer statistics, 2002. CA Cancer J Clin 55: 74-108, 2005.
7. Fisher B, Anderson S, Bryant J, et al: Twenty-year follow-up of a randomized trial comparing total mastectomy, lumpectomy, and lumpectomy plus irradiation for the treatment of invasive breast cancer. N Engl J Med 347: 1233-1241, 2002.

8. Jacobson JA, Danforth DN, Cowan KH, et al: Ten-year results of a comparison of conservation with mastectomy in the treatment of stage I and II breast cancer. N Engl J Med 332: 907-911, 1995.

9. Lacour J, Le M, Caceres E, Koszarowski T, Veronesi U and Hill C: Radical mastectomy versus radical mastectomy plus internal mammary dissection. Ten year results of an international cooperative trial in breast cancer. Cancer 51: 1941-1943, 1983.

10. Maddox WA, Carpenter JT, Laws HL, et al: A randomized prospective trial of radical (Halsted) mastectomy versus modified radical mastectomy in 311 breast cancer patients. Ann Surg 198: 207-212, 1983.

11. Turner L, Swindell R, Bell WG, et al: Radical versus modified radical mastectomy for breast cancer. Ann R Coll Surg Engl 63: 239-243, 1981.

12. Cabanas RM: An approach for the treatment of penile carcinoma. Cancer 39: 456-466, 1977.

13. Kim T, Giuliano AE and Lyman GH: Lymphatic mapping and sentinel lymph node biopsy in early-stage breast carcinoma: a meta-analysis. Cancer 106: 4-16, 2006.

14. Houssami N, Ciatto S, Turner RM, Cody HS and Macaskill P: Preoperative ultrasound-guided needle biopsy of axillary nodes in invasive breast cancer: meta-analysis of its accuracy and utility in staging the axilla. Ann Surg 254: 243-251, 2011.

15. Ko EY, Han BK, Shin JH and Kang SS: Breast MRI for evaluating patients with metastatic axillary lymph node and initially negative mammography and sonography. Korean J Radiol 8: 382-389, 2007.

16. Mameri CS, Kemp C, Goldman SM, Sobral LA and Ajzen S: Impact of breast MRI on surgical treatment, axillary approach, and systemic therapy for breast cancer. Breast J 14: 236-244, 2008.

17. Garcia Fernández A, Fraile M, Giménez N, et al: Use of axillary ultrasound, ultrasound-fine needle aspiration biopsy and magnetic resonance imaging in the preoperative triage of breast cancer patients considered for sentinel node biopsy. Ultrasound Med Biol 37: 16-22, 2011.

18. Harnan SE, Cooper KL, Meng Y, et al: Magnetic resonance for assessment of axillary lymph node status in early breast cancer: a systematic review and meta-analysis. Eur J Surg Oncol 37: 928-936, 2011.

19. Kvistad KA, Rydland J, Smethurst HB, Lundgren S, Fjosne HE and Haraldseth O: Axillary lymph node metastases in breast cancer: preoperative detection with dynamic contrast-enhanced MRI. Eur Radiol 10: 1464-1471, 2000.

20. Peare R, Staff RT and Heys SD: The use of FDG-PET in assessing axillary lymph node status in breast cancer: a systematic review and meta-analysis of the literature. Breast Cancer Res Treat 123: 281-290, 2010.

21. Valente SA, Levine GM, Silverstein MJ, et al: Accuracy of predicting axillary lymph node positivity by physical examination, mammography, ultrasonography, and magnetic resonance imaging. Ann Surg Oncol 19: 1825-1830, 2012.

22. Hwang SO, Lee SW, Kim HJ, Kim WW, Park HY and Jung JH: The comparative study of ultrasonography, contrast-enhanced MRI, and ${ }^{18}$ F-FDG PET/CT for detecting axillary lymph node metastasis in T1 breast cancer. J Breast Cancer 16: 315-321, 2013.

23. Quon A and Gambhir SS: FDG-PET and beyond: molecular breast cancer imaging. J Clin Oncol 23: 1664-1673, 2005.

24. Omoto K, Matsunaga $\mathrm{H}$, Take N, et al: Sentinel node detection method using contrast-enhanced ultrasonography with Sonazoid in breast cancer: preliminary clinical study. Ultrasound Med Biol 35: 1249-1256, 2009.

25. Aoki T, Moriyasu F, Yamamoto K, Shimizu M, Yamada M and Imai Y: Image of tumor metastasis and inflammatory lymph node enlargement by contrast-enhanced ultrasonography. World J Radiol 3: 298-305, 2011. 